\title{
Shear failure of AA2024 and AA7175 under high strain rate loading
}

\author{
Gunasilan Manar ${ }^{1,2}$, Patrice Longère ${ }^{1, *}$ \\ ${ }^{1}$ Université de Toulouse, ISAE-SUPAERO, ICA (CNRS 5312), Toulouse, France \\ ${ }^{2}$ National Defense University of Malaysia, Kuala Lumpur, Malaysia
}

\begin{abstract}
In the context of the design of aeronautical structures regarding accidental events, we are here investigating and comparing the shear failure of two aluminum alloys, namely AA2024 and AA7175, under high strain rate loading. With this aim in view, two experiments were carried out: (i) high strain rate shear compression of hat shaped structures, and (ii) impact test on the edge of double notched plates. The fractured surfaces of post-mortem specimens were observed using optical and scanning electron microscopes (SEM) in order to identify the failure mechanisms.
\end{abstract}

\section{Introduction}

Aeronautical structures have to withstand high strain rate overloading as encountered notably during impact loading, including e.g. hail storm or bird strike. In the context of the design of aeronautical structures regarding accidental events, it is thus of major interest to investigate the behavior of the constitutive materials under high loading rate.

Under high strain rate loading involving quasi adiabatic conditions, it has been shown that some high strength metallic materials may fail under a specifically dynamic shear localization mechanism known as adiabatic shear banding (ASB). ASB results from the competition between hardening and softening mechanisms and makes premature the ultimate fracture. ASB-assisted failure has been evidenced for high strength ARMOX500T steel [1], Ti-6Al-4V titanium alloy [2] as well as AA7075 aluminum alloy [3].

Aluminum alloys are widely used for primary aircraft structures due to their high specific mechanical properties and corrosion resistance. Aluminum alloys are also relatively cheap when compared with other metallic and composite materials in terms of manufacturing and maintenance cost, see [1]-[2]. AA2XXX and AA7XXX aluminum alloys are two of the most common series used for airframes, the former series (AA2XXX) having copper as the main addition element while the latter series (AA7XXX) has zinc as the main addition element [4].

We are here investigating and comparing the shear failure of two aluminum alloys, namely AA2024 and AA7175, under high strain loading. With this aim in view, two experiments were carried out: (i) high strain rate shear compression of hat shaped structures, and (ii) impact test on the edge of double notched plates. The fractured surfaces

\footnotetext{
* Corresponding author: patrice.longere@isae.fr
} 
of post-mortem specimens were observed using optical and scanning electron microscopes (SEM) in order to identify the failure mechanisms.

The experimental set-ups are briefly described in Section 2. The experimental results are presented and discussed in Section 3. Conclusions are given in Section 4.

\section{Experimental procedures}

The set-ups used for high strain rate shear compression of hat shaped structures and impact test on the edge of double notched plates are shown in Figs.1-2.
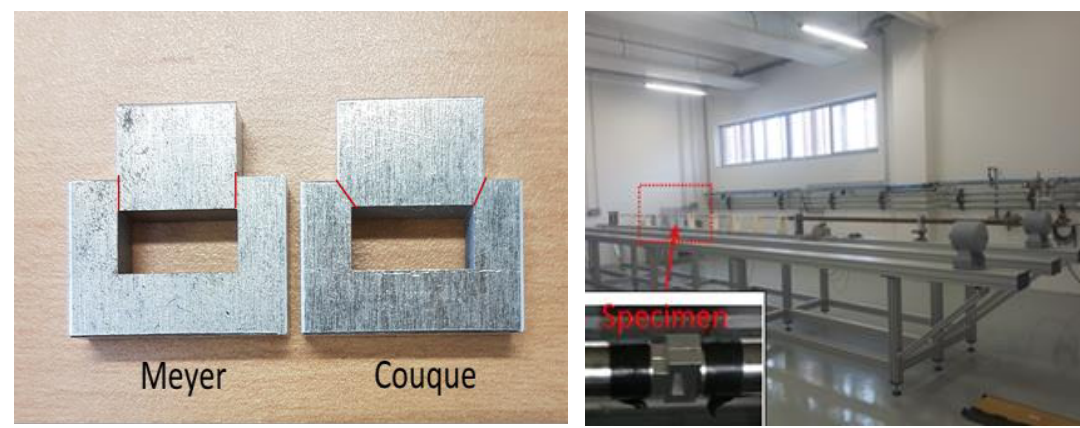

Fig. 1 High strain rate shear compression of hat shaped structures. Left: Geometries of the specimens. Right: Split Hopkinson pressure bar apparatus
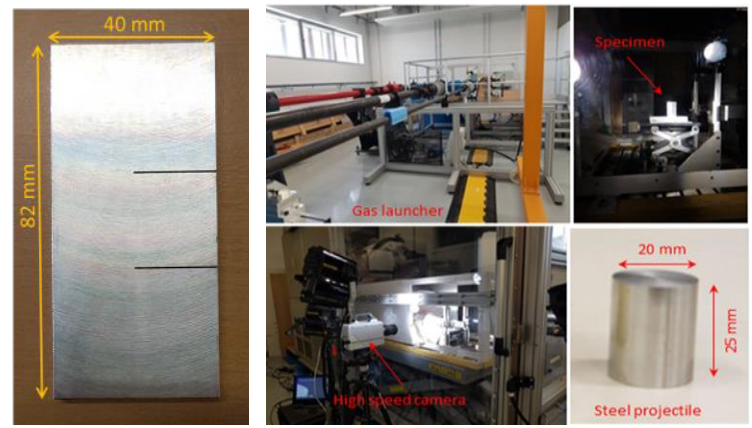

Fig. 2 Impact test on the edge of double notched plates. Left: Geometry of the plate. Right: Experimental set-up

Shear compression tests were conducted at ambient temperature and at high strain rate using a split Hopkinson pressure bar (SHPB) apparatus, see Fig.1. This apparatus consists in three steel bars which are striker, input and output bars. Input and output bars are equipped with strain gauges. The specimen to test is inserted between the input and output bars. The impact of the striker bar onto the input bar generates a compression wave which propagates throughout the input bar and loads the specimen. The parts of the incident wave reflected into the input bar and transmitted into the output bar are used to estimate the strain and stress in the specimen. The lengths of the striker, input and output bars are $0.3 \mathrm{~m}, 3 \mathrm{~m}$ and $2 \mathrm{~m}$ respectively with diameter of $20 \mathrm{~mm}$. Two geometries of hat shaped specimens (HSS) were used, namely Meyer [6] and Couque [7], see Fig.1. Meyer- and Couque-type HSS are designed in order to involve in the shear zone of interest (marked in red in Fig.1) a stress triaxiality ratio close to zero and negative, respectively.

Impact tests were carried out using a gas launcher, see Fig.2. The test consists in impacting 
a Kalthoff and Winkler [8] (KW)-type double-notched plate which simulates pre-crackcontaining structures, see Fig.2. Photron SA5 high-speed camera was used to record the projectile-plate interaction and crack advance during the impact loading at $10^{5}$ frames per second (fps) and 320 X 192 pixel $^{2}$ spatial resolutions. Lighting was provided by Dedolight HMI floodlight of 400W.

Post-mortem specimens were observed using optical and scanning electron microscopes (SEM).

\section{Results and discussion}

Results obtained for AA2024 and AA7175 aluminum alloys from high strain rate shear compression of hat shaped structures and impact test on the edge of double notched plates are presented and discussed in the following.

\subsection{High strain rate shear compression of hat shaped structures}

The force history curves obtained from the high strain rate shear compression tests are plotted in Fig.2 for the two aluminum alloys under consideration, viz. AA2024 and AA7175. According to Fig.2, the force is larger for Couque-type specimen compared to Meyer-type specimen for both materials, as a consequence of the stress triaxiality ratio (higher for Couque and lower for Meyer). On the other hand, whatever the specimen geometry, the force is higher for AA7175 compared to AA2024, as a consequence of the material strength (higher for AA7175 and lower for AA2024). At last, it can be seen for AA7175 that there is a drop in force from $\mathrm{t}=35 \mu \mathrm{s}$, whereas the force monotonically increases for AA2024.

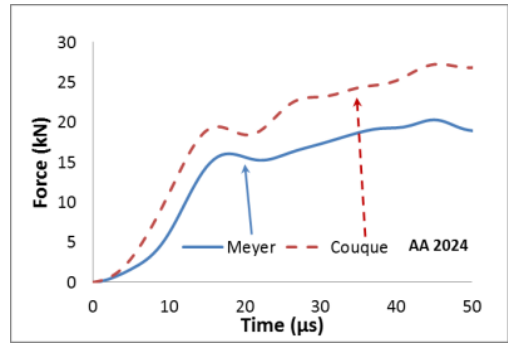

(a)

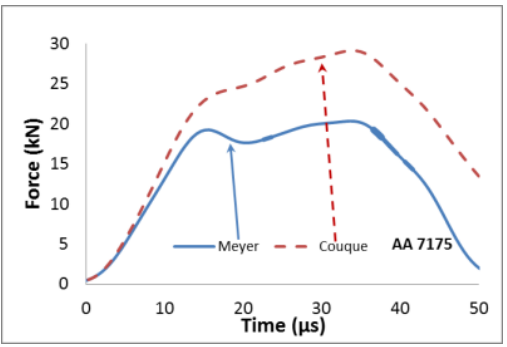

(b)

Fig. 2 Comparison of force history for AA2024 and AA7175 for different types of specimen geometry

Fig.3. shows the fractured surface of Couque-type specimen for AA2024. Two different zones can be evidenced on the surface. Microstructural observations shows flat shear surface for zone 1 and dimple cluster for zone 2, see Fig.4 (a) and (b).

Fig. 5 shows the fractured surface of Couque-type specimen for AA7175. One can distinguish three different zones on the fractured surface. Microstructural observations show flat shear surface for zone 1 and zone 2, see Fig. 6 (a) and (b). Zone 3 exhibits dimple cluster as depicted in Fig.6 (c). 


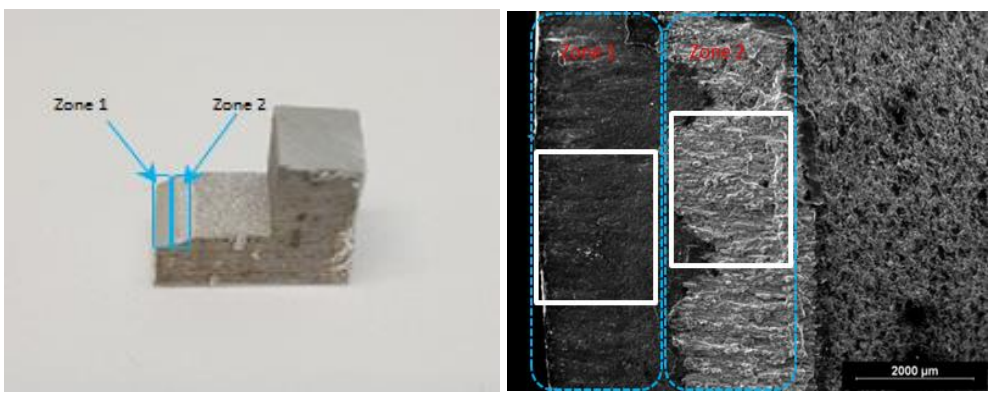

Fig. 3. Fractured surface of AA2024 for Couque type specimen

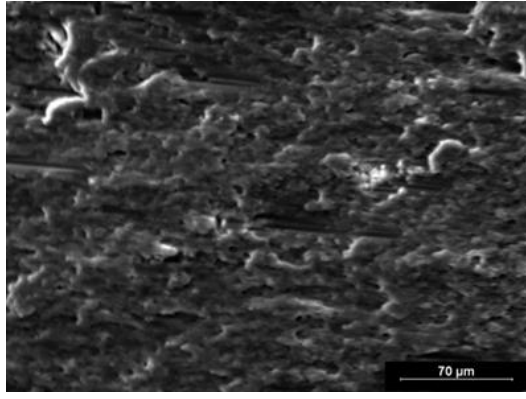

(a) Zone 1

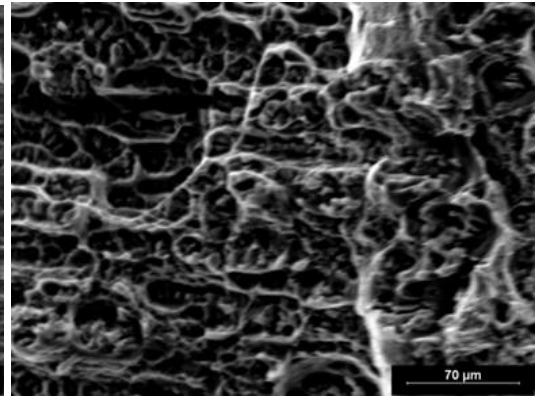

(b) Zone 2

Fig. 4. Microstructure of AA2024 for Couque type specimen for zone 1 and zone 2
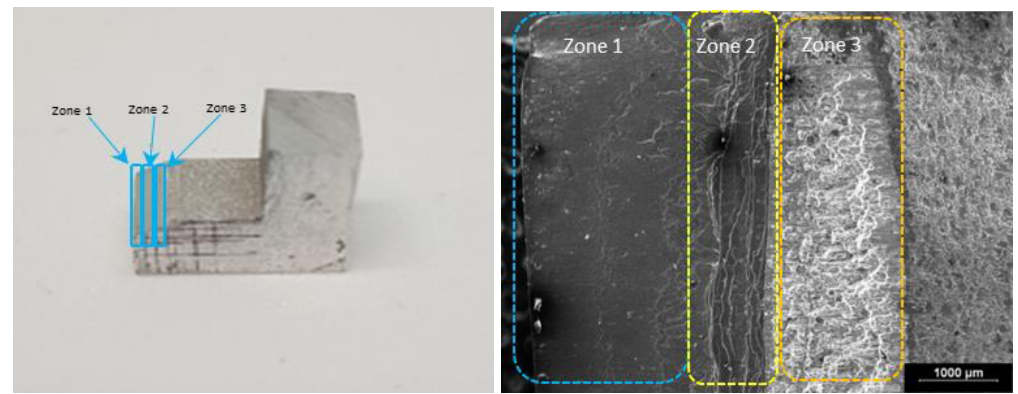

Fig. 5. Fractured surface of AA7175 for Couque type specimen

\subsection{Impact test on the edge of double notched plates}

Post-mortem double-notched plates made of AA2024 and AA7175 are shown in Fig.7 for different impact velocities of the projectile. It can be seen that the complete fracture occurs at different critical impact velocities depending on the alloy grade. The complete fracture results in crack propagation throughout the whole plate. The critical impact velocity for AA2024 is between 133 and 150 m.s. ${ }^{-1}$ whereas for AA7175 it is between 100 and $130 \mathrm{~m} . \mathrm{s}^{-}$ 1 , i.e. less. 


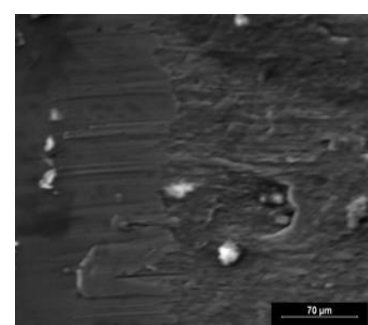

(a) Zone 1

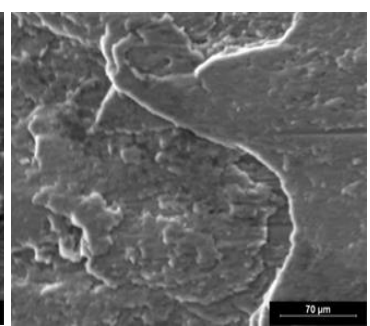

(b) Zone 2

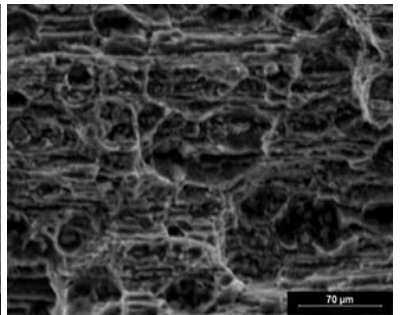

(c) Zone 3

Fig. 6 Microstructure of AA7175 for Couque type specimen for zone 1, zone 2 and zone 3

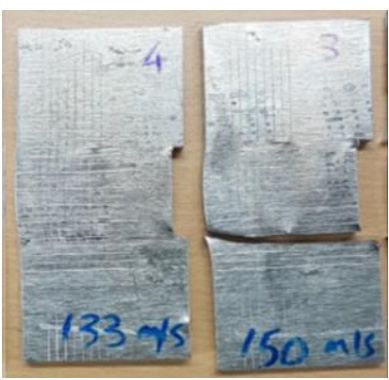

(a) AA 2024

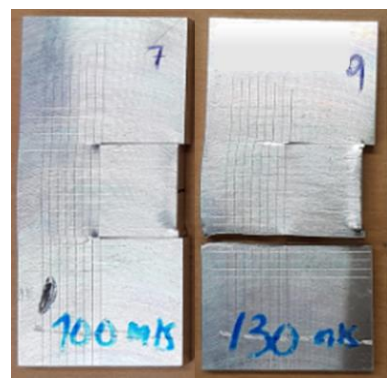

(b) AA 7175

Fig. 7 . KW specimens after impacted at different velocities

Fig. 8 (a) and (b) show the fractured surface of plates after impact at the velocities of 150 m.s ${ }^{-1}$ and $164 \mathrm{~m} \cdot \mathrm{s}^{-1}$ for AA2024 and AA7175 respectively. According to Fig. 8(a) two different fractured zones can be seen. Zone 1 shows the surface at crack initiation and zone 2 shows the surface at crack propagation which leads to fully fracture of the structure. Fig. 8 (b) also shows two zones where zone 1 corresponds to crack initiation and zone 2 to crack propagation until ultimate fracture. According to Fig.8 (b) crack lip area exhibits similarities with the crack initiation zone 1.

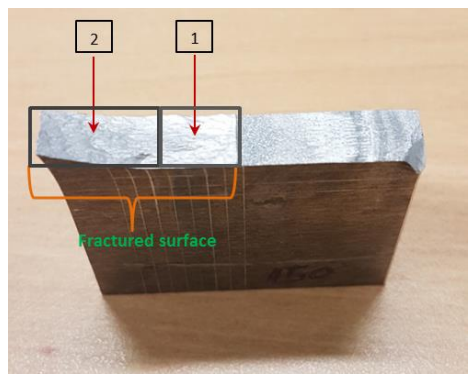

(a)

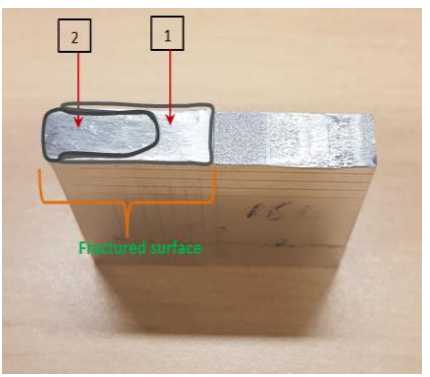

(b)

Fig. 8. Analysis of fractured surface (a) AA2024 after impact at $150 \mathrm{~m} / \mathrm{s}$ (b) AA7175 after impact at $164 \mathrm{~m} / \mathrm{s}$

Fig.9 shows the microstructure of AA2024 for both fracture zones after impact at $150 \mathrm{~m} . \mathrm{s}^{-1}$. Microstructural view for AA2024 shows that zone 1 is featured by flat shear surface (Mode II), see Fig. 9(a) and zone 2 is opening mode where microstructural view shows a dimple cluster (Mode I), see Fig. 9(b). 


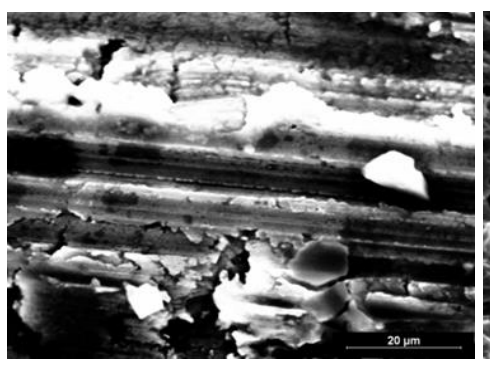

(a) Zone 1

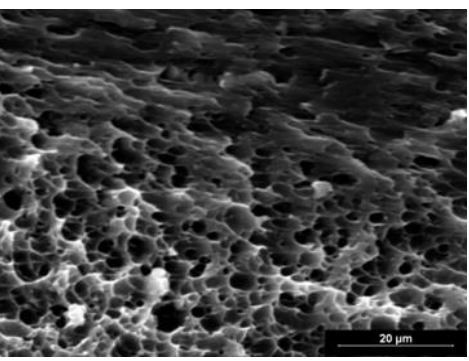

(b) Zone 2

Fig. 9. Microstructure view of AA2024 ruptured surfaces (a) Flat shear (b) Dimple cluster

Fig. 10(a) and (b) shows the microstructure of AA7175 after impact at $164 \mathrm{~m} \cdot \mathrm{s}^{-1}$ for zone 1 and zone 2 respectively. Microstructural view of AA7175 shows that both region are flat shear surface, hence the failure is mainly due to shear (Mode II). On the other hand, the observation of the crack lips reveals the presence of adiabatic shear bands, see Fig.11.

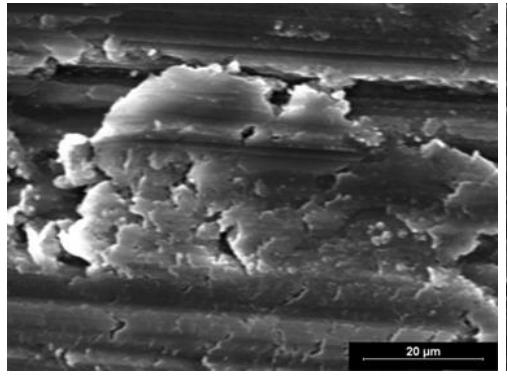

(a) Zone 1

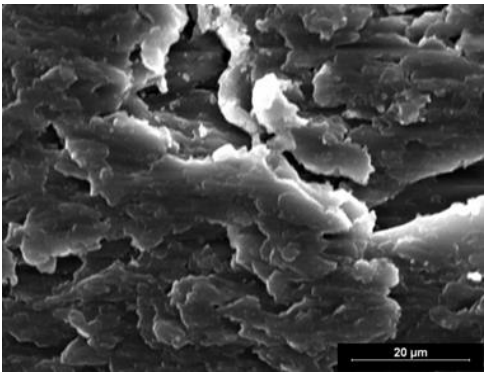

(b) Zone 2

Fig. 10.Microstructure view of AA7175 ruptured surfaces (a) Flat shear (b) Flat shear

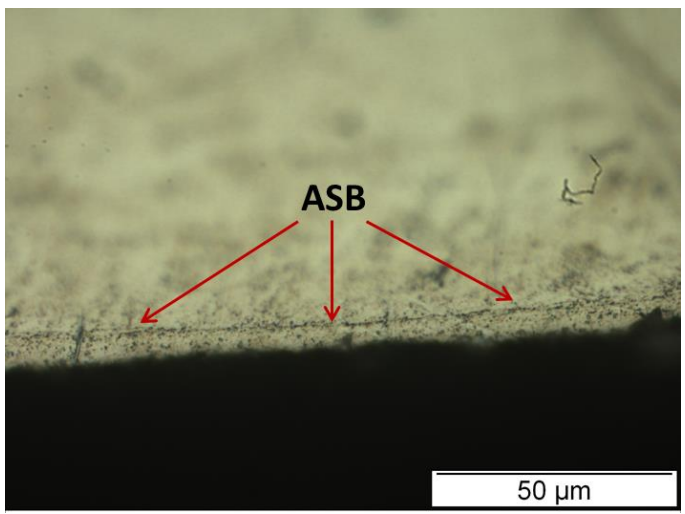

Fig. 11. Adiabatic shear band near crack lip of fractured surface for AA7175 


\subsection{Summary}

The results obtained from the dynamic shear compression tests show that the force is larger for Couque-type specimens compared to Meyer-type specimens, as expected. In terms of resistance, AA7175 shows larger force compared to AA2024 but exhibits a sudden drop in force as a consequence of a premature failure. Microstructural observation of Couque-shape specimen for AA2024 shows that the failure at early stage is due to shearing induced Mode II and at later stage the failure is predominantly controlled by tension induced Mode I. AA7175 Couque shape specimen shows three different regions. First and second region shows the failure is under shearing induced Mode II whereas the third region shows the tension induced mode I failure.

Impact tests show that the critical impact velocity at crack arrest for AA7175 doublenotched plates is close to $130 \mathrm{~m} \cdot \mathrm{s}^{-1}$ whereas for AA2024 it is close to $150 \mathrm{~m} \cdot \mathrm{s}^{-1}$, i.e. larger. Microstructural observation for AA2024 on fully fractured surface shows the early stage of fracture occurs under shearing induced Mode II and the latter stage was under tension induced Mode I whereas for AA7175 predominant by shearing induced Mode II failure and that shear localization has developed in the form of adiabatic shear bands.

\section{Concluding remarks}

High strain rate shear compression of hat shaped structures and impact test on the edge of double notched plates were carried out to investigate and compare the shear failure of AA2024 and AA7175 aluminum alloys under high loading rate. The experimental campaign has shown that while AA7175 possesses a higher strength it is less resistant to high rate shear loading than AA2024. Adiabatic shear banding observed on AA7175 (and not on AA2024) is the most likely reason for this premature failure.

\section{References}

1. E. Roux, P. Longère, O. Cherrier, T. Millot, D. Capdeville, and J. Petit, Mater. Des., vol. 75, pp. 149-159, (2015)

2. P. Longère and A. Dragon, Mech. Mater., vol. 80, no. PB, pp. 203-218, (2015)

3. C. Mondal, B. Mishra, P. K. Jena, K. Siva Kumar, and T. B. Bhat, Int. J. Impact Eng., vol. 38, no. 8-9, pp. 745-754, (2011)

4. Starke E.A. Jr and Staley J.T., Pergamon, vol. 32, pp. 131-172, (1996)

5. T. Dursun and C. Soutis, Mater. Des., vol. 56, pp. 862-871, (2014)

6. M. S. Meyer LW, Marcel Decker Inc, New York, (1986)

7. H.Couque, J. Phys. IV, vol. 110, pp. 423-428, (2003)

8. J. F. Kalthoff and S. Winkler, Proc. Int. Conf. Impact Load. Dyn. Behav. Mater., vol. 1, pp. 185-195, (1987) 